\title{
Privat: COVID-19 bei jugendlichen Athleten: Diagnose und Return to Sports
}

\section{EXERCISE IS MEDICINE}

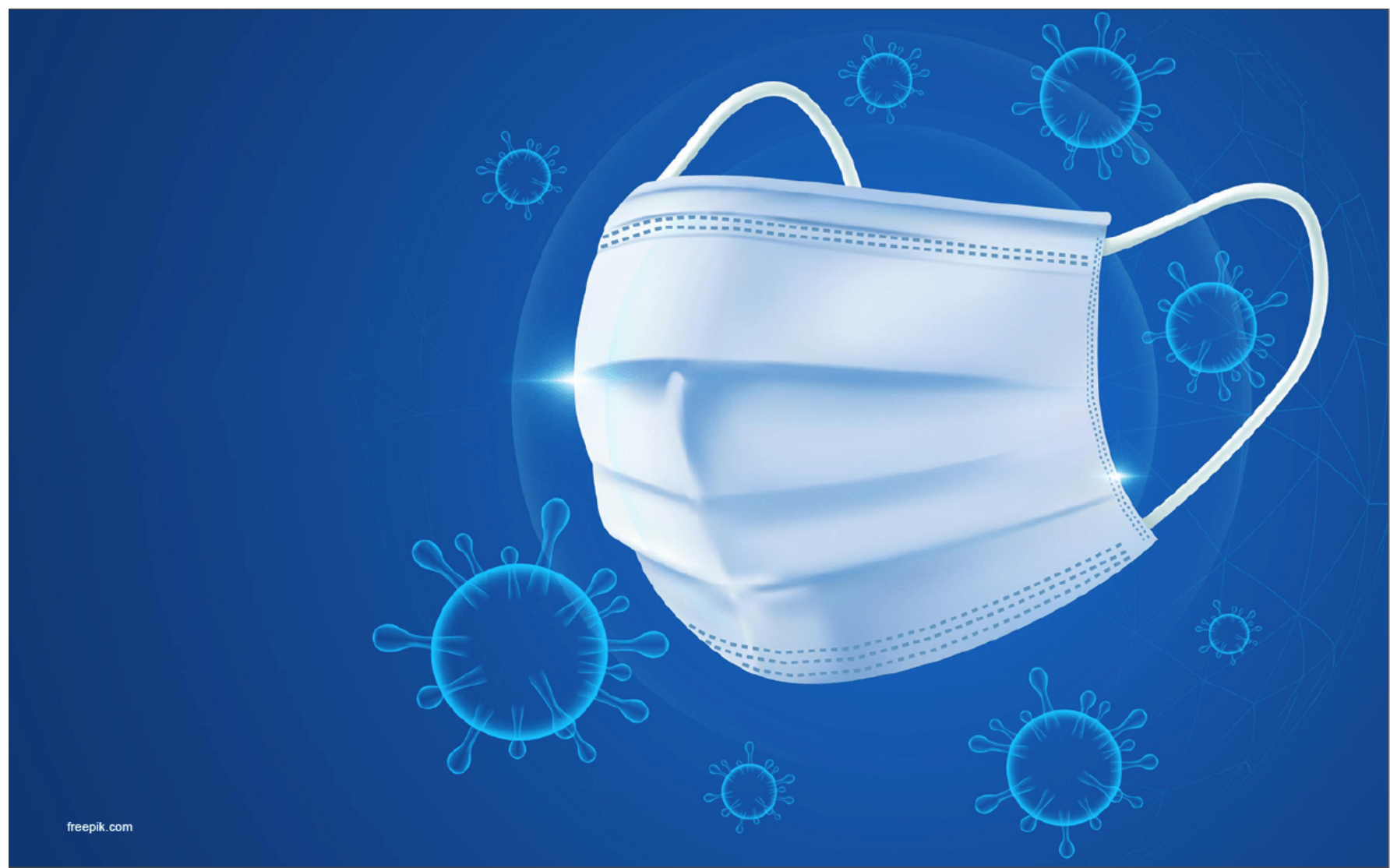

Kriemler Susi ${ }^{1}$, Siaplaouras Jannos ${ }^{2}$, Förster Holger ${ }^{3}$, Joisten Christine ${ }^{4}$

${ }^{1}$ Institut für Epidemiologie, Biostatistik und Prävention, Universität Zürich

- Präsidentin der Gesellschaft für Pädiatrische Sportmedizin

- Mitglied und Past-Präsidentin der Sport und Exercise Medicine Schwitzerland (SEMS)

${ }^{2}$ Praxis am Herz-Jesu-Krankenhaus Fulda

- Mitglied der Gesellschaft für Pädiatrische Sportmedizin

- Mitglied der Gesellschaft für Pädiatrische Kardiologie (DGPK)

- Vorstandsmitglied der Gesellschaft für Pädiatrische Sportmedizin

${ }^{3}$ Sport- und Kinderarztpraxis, Salzburg, Österreich

- Mitglied der Gesellschaft für Pädiatrische Sportmedizin

- Sportmedizinischer Beauftragter der Österreichischen Gesellschaft für Kinder- und Jugendheilkunde 
${ }^{4}$ IInstitut für Bewegungs- und Neurowissenschaft, Abteilung für Bewegungs- und Gesundheitsförderung, Deutsche Sporthochschule Köln

- Vorstandsmitglied der Österreichischen Gesellschaft für Sportmedizin und Prävention (ÖGSMP)

- Vizepräsidentin der Deutschen Gesellschaft für Sportmedizin und Prävention (DGSP)

\section{Summary}

Children and adolescents should be active in sports as much and often as possible. If permitted by the federal government and the states/cantons, this can take place now or, depending on the state, in the near future. Strict adherence to hygiene and distance rules and staying away from sport in the case of SARSCoV-2 typical symptoms can minimize the emergence of sources of infection and the transmission of the coronavirus. However, this is associated with a considerable psychological stress especially for children and adolscents. Although various diagnostic tests are available, the polymerase chain reaction (PCR) test is still considered the gold standard. However, a rapid antigen test can be a provisional procedure if time is of the essence and a PCR is postponed. The guidelines for an optimal "return to sports" should be based on the severity of the course after a corona infection and strictly adhered to because of the risk of myocarditis, which can potentially lead to sudden cardiac death.

\section{Zusammenfassung}

Kinder und Jugendliche sollen sportlich aktiv sein, und zwar so viel und oft wie möglich. Dies kann, falls vom Bund und den Ländern/Kantonen erlaubt, bereits jetzt oder je nach Land in naher Zukunft stattfinden. Das strikte Befolgen von Hygiene- und Distanzregeln und das Fernbleiben vom Sport bei SARSCoV-2-typischen Symptomen können das Entstehen von Infektionsherden und die Übertragung des Coronavirus minimieren, ist aber gerade bei Kindern und Jugendlichen mit einer grossen psychischen Belastung verbunden. Obwohl diverse diagnostische Tests zur Verfügung stehen, gilt der PolymeraseKettenreaktion-Test (polymerase chain reaction, PCR-Test) nach wie vor als Goldstandard. Ein AntigenSchnelltest kann jedoch ein provisorisches Vorgehen vorbahnen, wenn die Zeit drängt und eine PCR nachgeschoben wird. Die Richtlinien für einen optimalen Return to Sports sollen auf dem Schweregrad des Verlaufs nach einer Coronainfektion basieren und wegen des Risikos einer Myokarditis, welche potenziell zu einem plötzlichen Herztod führen kann, strikt eingehalten werden.

\section{Stellungnahme}

Dies ist eine Stellungnahme der Gesellschaft für Kindersportmedizin, einer internationalen Vereinigung zur Förderung von Bewegung und Sport im Kindes- und Jugendalter (GPS). Die Stellungnahme wurde in Zusammenarbeit mit Mitgliedern der Gesellschaft für Kindersportmedizin und der Deutschen Gesellschaft für Sportmedizin und Prävention, der Österreichischen Gesellschaft für Sportmedizin und der Sport \& Exercise Medicine Switzerland (SEMS) erstellt. 


\section{Bedeutung von Sport und Bewegung während der COVID-19-Pandemie}

Die Coronapandemie hat das Leben von Kindern und Familien verändert, auch hinsichtlich der Sportaktivität. Diese Stellungnahme soll Ärzten helfen, das Risiko der Ausbreitung von SARS-CoV-2 innerhalb der Familien und bei Sportaktivitäten zu reduzieren, und beinhaltet einen Leitfaden für die Rückkehr in den Sport nach COVID-19 für Kinder und Jugendliche bis 16 Jahre. Bis auch Kinder und Jugendliche flächendeckend gegen SARS-CoV-2 geimpft sein werden, gilt es dabei stets eine optimale Risiko-Nutzen-Strategie zu überdenken.

Die Bedeutung von Bewegung und Sport für Kinder und Jugendliche ist immens und unbestritten und reicht von körperlichem Gewinn (kardiovaskuläre Gesundheit, Kraft, Körperzusammensetzung, Fitness etc.), über psychisch stabilisierende, stimmungsaufhellende Auswirkungen, zu einer besseren Sozialisierung und zum Erlernen von exekutiven Funktionen wie Impulskontrolle, Konzentration und Gedächtnis (WHO guidelines on physical activity and sedentary behaviour). Junge Menschen sind von der Coronakrise betroffen mit unbestrittenen Auswirkungen auf die psychische Gesundheit, die körperliche Fitness, ihre sozialen Beziehungen und schlussendlich auch ihre Entwicklung (UNICEF - The impact of COVID-19 on mental health of youth). Bewegung und Sport bieten gerade in dieser Phase der Coronabedingten Einschränkungen Möglichkeiten, auf allen Ebenen positiv zu wirken: Spass, Bewegung und zwischenmenschliche Beziehungen können unter Gleichaltrigen gelebt werden und damit die grossen Herausforderungen der Entwicklung und des Erwachsenwerdens bewerkstelligt werden.

\section{Risiko von Sport während der COVID-19-Pandemie}

Obwohl noch viel Wissen hinsichtlich COVID-19 bei Kindern und Jugendlichen fehlt, gibt die Mehrzahl der wissenschaftlichen Daten folgendes Bild wieder: Kinder infizieren sich gleich häufig wie Erwachsene, sie zeigen jedoch häufig einen asymptomatischen oder milden symptomatischen Verlauf, schwere Infektionen sind sehr selten, hospitalisiert werden lediglich 0,1\%, Todesfälle kommen in weit unter 0,1\% vor [1]. LongCOVID und das PIMS-Syndrom (Pediatric Inflammatory Multisystem Syndrome) sind selten bei Kindern, aber wenn diese Folgen einer SARS-CoV-2-Infektion auftreten, können sie das Leben der Kinder stark beeinträchtigen, im letzteren Fall gar stark gefährden [2,3,4]. Die Übertragung des Virus unter Kindern und auf Erwachsene, insbesondere wenn asymptomatisch, kommt zwar vor, wurde aber in der Vergangenheit relativ selten beobachtet [5,6]. Bisher waren auch Ausbrüche in Schulen ebenfalls selten [7,8]. Dies kann sich durchaus ändern in naher Zukunft mit dem Auftreten von neuen Varianten. Da die Impfung für Kinder unter 12 Jahren noch nicht erlaubt ist, wird uns die Coronainfektion bei Kindern in den kommenden Monaten noch beschäftigen.

Der enge Kontakt mit einer infizierten Person ist der primäre Übertragungsweg. Jugendliche scheinen das Coronavirus gleich häufig wie Erwachsene zu übertragen [5,6,9,10], während Kinder bisher als weniger ansteckend galten [6]. Mit der Dominanz der infektiöseren Delta-Variante und dem fehlenden Impfschutz bei den Kindern kann sich dieses Bild ändern. Bei sportmedizinischer Betrachtung fliessen neben dem Alter weitere Faktoren wie Sportart (Anzahl Spieler, Abstand, Häufigkeit und Dauer der engen Kontakte), Umgebungsbedingungen (indoors versus outdoors, Dimension der Gebäude, Lüftung) sowie Übertragungsmöglichkeiten über Material und Oberflächen (Bälle, geteilte Ausrüstung, Oberflächen und Räume) mit in die Risikobeurteilung ein (https:// http://www.ecdc.europa.eu/en/covid-19/latest-evidence/transmission). 


\section{Nutzen-Risiko-Abwägung von Bewegung und Sport}

Die Entscheidung über die Beteiligung im Sport bzw. die Rückkehr in den Sport nach einer COVID-19Infektion soll das Risiko gegenüber dem Nutzen in die Waagschale legen. Dazu gehören individuelle Begebenheiten und Impfstatus, aber insbesondere inwiefern der sporttreibende Athlet oder seine Familie bei Ansteckung ein Risiko für einen schweren Verlauf einer Coronainfektion tragen. Die Wahrscheinlichkeit einer Infektion kann je nach Sportart reduziert, aber nicht eliminiert werden. Die Entscheidung, ob den Kindern und Jugendlichen eine Sportaktivität erlaubt wird, muss nach Beratung durch Sportmediziner von den Eltern getragen werden. In der grossen Mehrheit der Fälle sind Kinder und Jugendliche nicht schwer betroffen und sollten nicht unnötig in ihrer sportlichen Aktivität eingeschränkt werden.

\section{Testen von SARS-CoV-2}

Das Testen von SARS-CoV-2 vor der Teilnahme im Sport wird bei vorhandenen Symptomen empfohlen, wenn ein relevanter Kontakt zu einer infizierten Person stattgefunden hat oder wenn Empfehlungen von Seiten der Gesundheitsministerien oder Sportverbände bestehen (siehe Websites Bund, Länder/Kantone, nationale pädiatrische Gesellschaften). In den DACH-Ländern werden die Weisungen laufend angepasst, sodass wir hier auf die Beschreibung des Vorgehens verzichten und auf die relevanten Websites verwiesen wird: Österreich; Deutschland; Schweiz. Wir empfehlen generell, Testungen wann immer möglich grosszügig durchzuführen, idealerweise mit gepoolten und technisch einwandfrei durchgeführten PCRTests. Wenn das Virus nicht auf dem Wattestäbchen sitzt oder ausgespuckt wird, kann auch ein PCR-Test falsch negativ sein. Es gilt ebenfalls zu beachten, dass es sich auch bei getesteten Personen nur um eine Momentaufnahme handelt, und dass vor allem Schnelltests, insbesondere bei asymptomatischen Kindern und Jugendlichen, oft falsch negativ sind (bis zu 60\%!) [11]

\section{Präventive Massnahmen zur Verhinderung einer Ansteckung}

Hohe aktuelle Ansteckungszahlen auf Gemeindeebene erhöhen das generelle Risiko einer Ansteckung von jugendlichen AthletInnen, TrainerInnen und Familien. Wie vorsichtig mit einer Öffnung umgegangen wird, hängt von den generellen Ansteckungszahlen, dem Prozentsatz der Durchimpfung, und dem Verlauf und den geltenden Inzidenzgrenzwerten ab. Diverse länderspezifische Websites geben darüber Auskunft und werden hier nicht einzeln thematisiert, da sie kontinuierlich angepasst werden (Österreich, Deutschland, Schweiz). Diese Massnahmen können einfacher und vollständiger durchgesetzt werden, wenn in den Vereinen jeweils ein medizinischer und ein organisatorischer Verantwortlicher definiert und offiziell ernannt werden. Der organisatorische Mitarbeiter ist für die Durchsetzung der präventiven Massnahmen verantwortlich, der medizinische Verantwortliche übernimmt alle medizinischen Belange inklusive Testung, Diagnostik und Kontakt zu den Verantwortlichen des Contact Tracings. Geimpfte Jugendliche haben einen vollen Impfschutz ab 2 Wochen nach der 2. Impfung. Dies bedeutet, dass Jugendliche nach der 1. Impfung oder im Fenster bis 2 Wochen nach der 2. Impfung noch nicht vollständig gegen eine Ansteckung geschützt sind und die Weisungen entsprechend den Ungeimpften befolgen sollten.

Eine Impfung gegen SARS-CoV-2 ist für Jugendliche ab 12 Jahren zugelassen und bietet in der Mehrheit 
einen Schutz vor einer Ansteckung mit dem Virus mit einer minimalen Nebenwirkungsrate [12], welche viel seltener sind als die Nebenwirkungen der Infektion selbst [13]. Geimpfte Jugendliche haben wie auch Erwachsene einen vollen Impfschutz ab 2 Wochen nach der 2. Impfung. Dies bedeutet, dass Jugendliche nach der 1. Impfung oder im Fenster bis 2 Wochen nach der 2. Impfung noch nicht vollständig gegen eine Ansteckung geschützt sind und die Weisungen entsprechend den Ungeimpften befolgen sollten. Auch diesbezüglich können Weisungen in den verschiedenen Ländern variieren und sollten beachtet werden. Bei jeder Sportaktivität mit ungeimpften Kindern und Jugendlichen vermindern die nachfolgenden Massnahmen das Risiko einer Infektion. Diese sollen, wann immer möglich, beachtet werden:

- Sofortiges Fernbleiben von Training und Wettkämpfen bei Symptomen. Der Trainer kann proaktiv vor jedem Training nach Symptomen fragen.

- Testung bei Symptomen und engem Kontakt zu einer infizierten Person.

- Trainingseinheiten und Wettkämpfe möglichst draussen durchführen. Aktivitäten im Freien vermindern das Ansteckungsrisiko. Sollten Aktivitäten im Freien nicht möglich sein, muss auf eine regelmässige Lüftung und Filtration der Luft geachtet werden.

- Nutzung von engen, nicht zu lüftenden Räumen (Kraftraum) meiden bzw. minimieren.

- Regelmässiges Stosslüften.

- Möglichst Einhalten von Distanzregeln. Kontakte mit Distanz von <2 m über 15 Min. oder mehr pro Tag erhöhen das Infektions- und Übertragungsrisiko. Das Nichteinhalten dieser Regeln muss auf ein Minimum beschränkt werden.

- Minimierung von direktem Körperkontakt. TrainerInnen können das Training anpassen bei Sportarten, bei denen die Distanzregel von 2 Metern nicht eingehalten werden kann wie bei Judo, Basketball, indem mehr auf ein individuelles Aufbautraining gesetzt wird oder in konstanten Kleingruppen gearbeitet wird.

- Sport in möglichst gleichbleibenden Kleingruppen.

- Reduktion von Singen, Rufen, Schreien, vor allem in Innenräumen.

- Reduktion von hochintensiven Aktivitäten in Innenräumen. Hochintensive Aktivitäten erhöhen das Risiko einer Übertragung durch eine vermehrte Aerosolbildung, insbesondere wenn sie in schlecht belüfteten Innenräumen stattfinden. Sie sollten draussen unter Einhaltung von Distanz durchgeführt werden.

- Maskenschutz vor allem bei Gruppenbildung und Missachtung der Abstandsregeln in Innenräumen. Änderungen sind zu beachten aufgrund der aktuellen Websites der betreffenden Länder:

- Österreich

- Deutschland

- Schweiz

- Strikte Hygienemassnahmen.

AthletInnen und TrainerInnen sollten zu häufigem Händewaschen mit Seife über mindestens 20 Sekunden oder zur Händedesinfektion angehalten werden, wann immer dies möglich und nötig ist. Sollte jemand in die Hände niesen, soll eine sofortige Händedesinfektion stattfinden.

- Regelmässige (mindestens einmal täglich bzw. nach Gebrauch) Desinfektion von Flächen, die regelmässig gebraucht werden (Garderoben, Toiletten, Wasserhähne, Türklinken usw.) Minimierung von gemeinsamem Gebrauch von Sportgeräten oder zumindest regelmässige Desinfektion von Geräten und Flächen, die gemeinsam gebraucht werden.

- Kein Austausch von Essen und Getränken (Trinkflaschen).

- Minimale Reisetätigkeit von TrainerInnen und SportlerInnen.

- Notfallplan bei Verdacht auf eine SARS-CoV-2-Infektion innerhalb der Sportteams. Vorhandensein von Notfallnummern und einem klaren Plan von Isolations-/Quarantänemassnahmen 
bei Verdacht auf eine SARS-CoV-2-Infektion. Dazu gehören eine sofortige Isolation des möglichen Indexfalls, Quarantäne aller SpielerInnen, die in engem Kontakt ( $<2$ m über 15 Min.) waren und die Information der betreffenden Gesundheitsbehörden, welche das weitere Prozedere festlegen.

\section{Diagnostik}

Der Wert eines routinemässigen Testens auf COVID-19 ist vor der Teilnahme an einer Sportveranstaltung, sei es Training oder Wettkampf, nicht belegt. Entsprechende Tests sind in jedem Fall indiziert, wenn ein Athlet symptomatisch ist, sie/er in engem Kontakt mit einer infizierten Person war oder eine Testung von den Behörden gefordert wird. Die Testung von SARS-CoV-2-Antikörpern ist je nach Land empfohlen.

Indikationen zur Testung sind also:

- Athlet mit COVID-19-typischen Symptomen (unabhängig einer Impfung gegen SARS-CoV-2)

- aFieber von $38-5^{\circ} \mathrm{C}$ oder mehr oder Schüttelfrost

- -Husten

- पVerstopfte oder fliessende Nase

- QVerlust des Geschmacks- oder Geruchsinns

- $\square$ Atembeschwerden, Atemnot

- -Schmerzen in Muskeln oder Gelenken

- -Müdigkeit oder Kopfschmerzen

- -Halsschmerzen/Halskratzen

- 口Übelkeit, Erbrechen oder Durchfall

- Ein Athlet mit engem Kontakt zu einer SARS-CoV-2-positiv getesteten Person wird definiert durch ein Nichteinhalten der Distanzregel über mehr als 10 bis 15 Min. Dauer (siehe auch länderspezifische Regelungen).

- Athleten mit Empfehlung für ein Screening (Schule, Arbeitsplatz, Wettkampf).

- Vorgabe der Behörden.

\section{Art der Testung}

Es gibt aktuell drei verschiedene Testverfahren: Polymerase chain reaction (PCR), Antigen- und Antikörper-Tests. Der PCR-Test ist aktuell der Goldstandard für den Nachweis von SARS-CoV-2 bei Kindern und Erwachsenen. Die Entnahme erforderte in der Vergangenheit einen Nasen-Rachen-Abstrich, neuerdings gilt Speichel/Rachenspülwasser oder ein buccaler Abstrich als valide und verhindert eine schlechte Erfahrung durch einen unangenehmen Nasen-Rachen-Abstrich [14]. Um gültig zu sein, muss der PCR-Test aber offiziell unter direkter Aufsicht einer Fachperson abgenommen werden. Die Analysen werden in einem speziellen Labor durchgeführt, ein Testresultat ist frühestens nach vier Stunden, meist am nachfolgenden Tag zu erwarten. Inzwischen führen ausgewählte Labors automatisch Surrogat-PCRs zur Detektion möglicher Virusmutationen durch.

Ein Antigen-Test führt zwar innerhalb von 15-30 Minuten zum Resultat, hat allerdings eine schlechtere Sensitivität. Dies bedeutet, dass mehr falsch negative Resultate auftreten als bei einer PCR-Untersuchung, vor allem in sehr frühen oder späteren Krankheitsstadien und bei asymptomatischen Kindern und Jugendlichen. Positive Testresultate sind meist reliabel, aber falsch positive Tests kommen dennoch vor, insbesondere wenn die SARS-CoV-2-Prävalenz tief ist. Antigen-Tests sind ideal bei Vorhandensein von 
Sports \& exercise medicine switzerland

Symptomen, wenn der Zeitfaktor eine Rolle spielt (Schule, Trainingslager, Wettkämpfe) und ein PCR-Test sofort nachgeschoben werden kann. Bei positivem Antigen-Test besteht bis zum Resultat des PCR-Tests Isolationspflicht.

Antikörper-Tests messen keine akuten Infekte, sondern geben Hinweise auf eine früher durchgemachte Infektion. Sie finden in der Akutdiagnostik deshalb keine Anwendung. Über die sich in der Zulassung befindenden oder bereits per Sonderzulassung zugelassenen «Selbsttests» lässt sich aktuell noch nicht abschliessend urteilen. Möglicherweise helfen sie trotz geringerer Sensitivität und Spezifität durch ihre Screeningfunktion, das Infektionsgeschehen einzudämmen. 


\section{COVID-19 verhindern - diagnostizieren - return to sports} für Kinder und Jugendliche

\section{verhindern}

Sofortiges Fernbleiben von Training und Wettkämpfen bei Symptomen!

Exposition:

- Sport möglichst im Freien

- bei Indoorsport regelmässiges Stosslüften

- Einhalten von Distanzregeln ( $2 \mathrm{~m})$

- Verhinderung/Minimierung von direktem Körperkontakt

- Sport in gleichbleibenden Kleingruppen

- kein Austausch von Essen und Getrānken

- möglichst keine ungeimpften Gāste

Hygienemassnahmen:

- Regelmāssige Desinfektionsmassnahmen

- Tragen von Masken, siehe jeweilige

Bestimmungen der Länder:

Deutschland

Österreich

$\underline{\text { Schweiz }}$

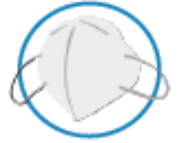

Notfallplan bei Verdacht auf eine COVID-19- Infektion innerhalb des Sportteams

- Beachtung offizieller aktueller Regelungen bzgl. Training und Wettkampf

nicht empfohlen wird das Tragen einer Maske in Situationen, in denen hierdurch eine besondere Gefährdung entstehen kann: z.B. Kunstturnen, rhythmische Gymnastik, Kampfsport mit Kōrperkontakt
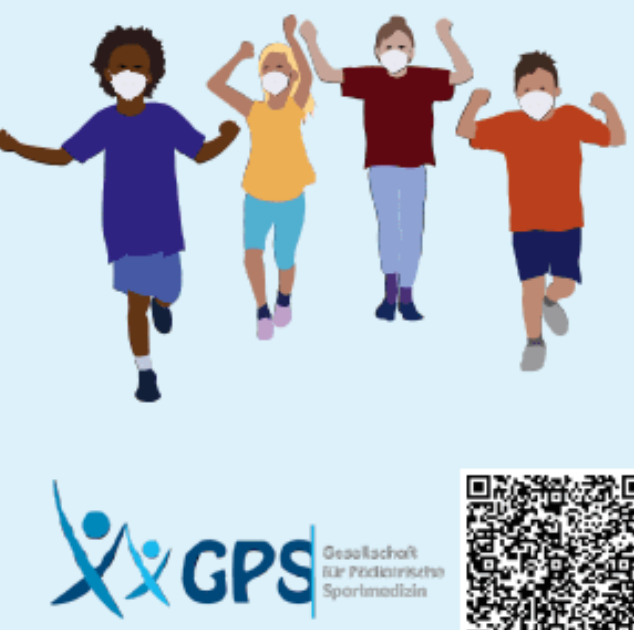

\section{diagnostizieren}

\section{wen testen?}

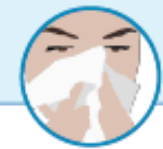

-Athlet/innen mit COVID-19 Symptomen

- asymptomatische Kontaktpersonen nach Richtlinien des Landes ${ }^{12,3}$

-Athleten/innen mit Empfehlung für ein Screening (Schule, Verein, Wettkampf)

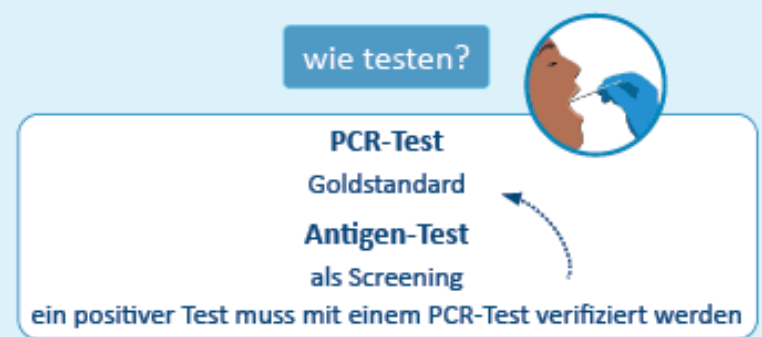

asymptomatisch/ leichte

Erkrankung

$<3$ Tage Fieber $>38.5^{\circ} \mathrm{C}$ Myalgien, ORL-, GI-Symptome
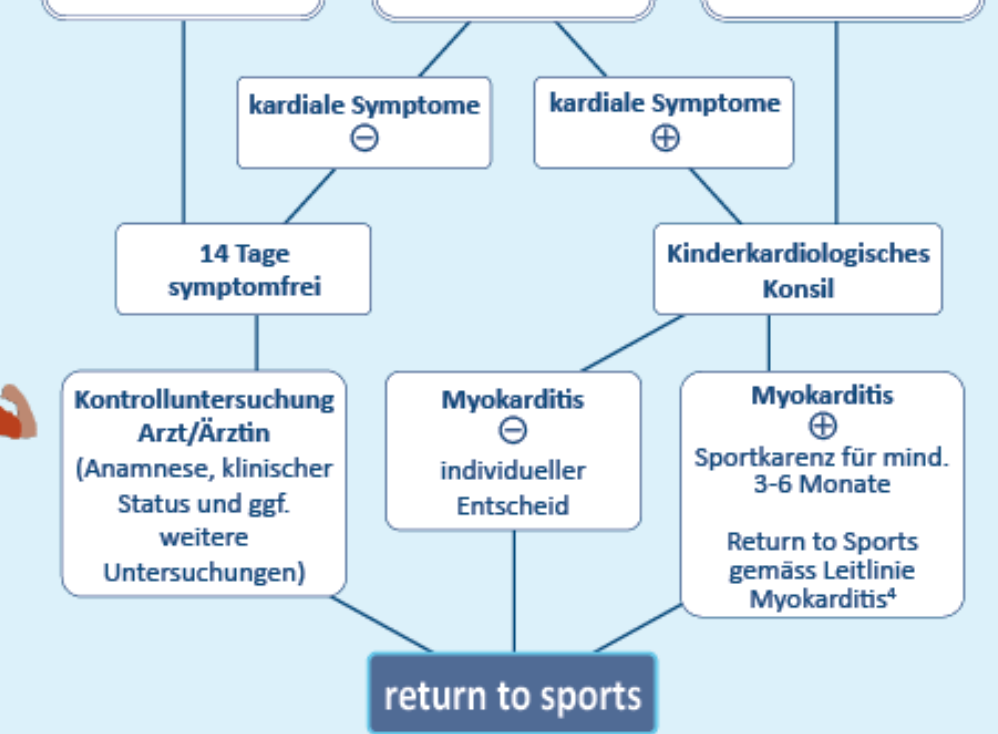

${ }^{1}$ Richtlinie Österreich: Was Kontaktpersonen wissen müssen

${ }^{2}$ Richtlinie Schweiz: BAG Umgang mit Fallen und ihrem Kontakten BAG COVID-19 und Sport 'Richtlinien Deutschland: Empfehlungen des RKI für das Management von Kontaktpersonen.. "DGPK Leitlinie Myokarditis

Zur ausführlichen Stellungnahme und weiterführenden Informationen gelangen Sie hier. 


\section{Return to Sports}

Es existieren zahlreiche Empfehlungen zum Return-to-Sports-Thema für den erwachsenen Athleten $[15,16]$, aber nur vereinzelt für Kinder und Jugendliche [17,18]. Aus verschiedenen Gründen scheint es inadäquat, die Empfehlungen der Erwachsenen auf Kinder und Jugendliche zu übertragen. Pädiatrische SARS-CoV-2-Infektionen laufen meist asymptomatisch oder mit milden Symptomen ab. Diese milden Verläufe sind die Norm und implizieren grundsätzlich keine speziellen Massnahmen über die üblichen Quarantäneregeln hinaus. Es gilt jedoch zu beachten, dass in seltenen Fällen eine Myokarditis auch bei asymptomatischen AthletInnen mit milden Symptomen vorkommen kann. Pathologische MRI-Befunde wurden in bis zu 46\% von SARS-CoV-2-infizierten AthletInnen gefunden [19]. Oft haben diese Befunde jedoch keine pathologische Bedeutung, überlappen mit einer gesunden Kontrollpopulation und nur in wenigen Fällen findet sich eine Myokarditis [13,20]. Mässig schwere oder schwere Verläufe sind selten, verlangen aber eine sorgfältige sportmedizinische Untersuchung mit Einbezug eines pädiatrischen Kardiologen, um eine Myokarditis frühzeitig auszuschliessen. Im Falle eines pädiatrischen multisystemischen inflammatorischen Syndroms (PIMS-TC oder MIS-C) muss zwingend ein multidisziplinäres Team involviert werden.

Das kardiologische Workup im Kindesalter muss verschiedene Faktoren beachten: Es stützt sich wie bei Erwachsenen auf EKG, Echokardiologie, Belastungstests und Troponin in der Beurteilung zur Freigabe zum Sport. Es besteht eine grosse Variabilität in der Intensität, mit welcher der Sport betrieben wird. Ein achtjähriger Breitensport-Fussballer soll liberaler behandelt werden als ein 16-jähriger Basketballer, der internationale Wettkämpfe bestreitet. Die Return- to-Sports-Frage ist wichtig, da trotz allem die Gefahr einer kardialen Schädigung oder Myokarditis ausgeschlossen werden muss, da Letztere, wenn auch selten, zum plötzlichen Herztod bei jungen AthletInnen führen kann [21] und deshalb mit grösster Sorgfalt auszuschliessen ist [22]. Schlussendlich kann kein Arzt das Vorhandensein von geschädigten Myozyten und somit eine kardiale Beteiligung der SARS-CoV-2-Infektion gänzlich ausschliessen. Deshalb soll sichergestellt werden, dass die AthletInnen die Symptome einer Myokarditits (Thoraxschmerzen, Atemnot, Leistungslimitierung, Pulsunregelmässigkeiten) kennen und bei Bedarf ein Notfallplan perfekt funktioniert mit kardiopulmonaler Wiederbelebung, Defibrillator und Notfallmedikamenten. In die ärztliche Beurteilung soll einfliessen, wann die SARS-CoV-2-Infektion stattgefunden hat, wie stark diese verlaufen ist und welche Sportart wie intensiv vom Kind oder Jugendlichen betrieben wird:

1. SARS-CoV-2-positiv ohne oder mit milden Symptomen ( $<3$ Tage Fieber von $38,5^{\circ} \mathrm{C}$ oder mehr, kurze Dauer von Myalgien, Müdigkeit, Symptome der oberen Luft- und Atemwege [ORL] oder gastrointestinal) Dies ist der am häufigsten vorkommende «Normalfall». Mindestens 10-14 Tage ab Symptombeginn und/oder positivem PCR+ soll kein Kontakt mit anderen Kindern und kein Sport stattfinden (diese Richtlinien werden immer wieder angepasst und sollten bei Bedarf in jedem Land separat nachgeschaut werden). Eine eingehende körperliche Untersuchung soll auf jeden Fall durchgeführt werden. Bei Symptomfreiheit und einwandfreiem Befund kann der Sportler wieder zurück in den Sport mit langsamem Aufbau. Einem vollkommen asymptomatischen Sportler kann durchaus erlaubt werden, zu Hause ein leichtes Training, zum Beispiel auf dem Fahrradergometer, schon während der Rekonvaleszenz aufzunehmen. Symptome wie Brustschmerzen, Herzstechen, inadäquate Atemnot oder Schweissneigung, Herzstolpern oder -rasen, Schwindelanfälle oder Synkopen können in seltenen Fällen vorkommen und Hinweis auf eine kardiale Beteiligung sein. Bei geringstem Verdacht muss zwingend eine kardiologische 
Abklärung beim Spezialisten erfolgen, insbesondere beim Leistungssport betreibenden Jugendlichen. Das EKG ist typischerweise abnormal bei einer Myokarditis [23]. Rückführung in den Sport kann erfolgen bei vollkommener Beschwerdefreiheit und keinerlei Einschränkungen in normalen täglichen Aktivitäten. Idealerweise geschieht dies durch einen progressiven Return to Sports über ein Minimum von sieben Tagen nach Symptomfreiheit von zwei Wochen.

2. SARS-CoV-2-positiv mit mässig starken Symptomen ( $\geq 3$ Tage Fieber $38,5^{\circ} \mathrm{C}$ und mehr, Myalgien, Lethargie) und/oder leichter Pneumonie ohne Sauerstoffbedarf oder Hospitalisation ausserhalb Intensivstation Eine Konsultation bei einem Kinderarzt oder pädatrischen Kardiologen mit EKG und weiteren kardiologischen Abklärungen ist indiziert frühestens ab zehn Tagen nach positivem Testresultat und Symptomfreiheit. Return to Sports erfolgt nach Richtlinien des Kardiologen. Diese Patienten können eine subklinische Myokarditis durchlaufen, auch wenn die Abklärungen normal sind/waren. Rückführung in den Sport bei vollkommener Beschwerdefreiheit und keinerlei Einschränkungen in normalen täglichen Aktivitäten und einem normalen EKG im Verlauf, bei Indikation auch mit Bestimmung von Troponin und Echokardiografie. Bei jüngeren Patienten unter 12 Jahren, insbesondere wenn die Sportausübung weniger intensiv ist, sind diese Massnahmen nicht zwingend notwendig. Ein progressiver Return to Sports erfolgt über ein Minimum von 7-14 Tagen nach Symptomfreiheit von mindestens zwei Wochen.

\section{SARS-CoV-2-positiv mit starken Symptomen und meist myokardialer Beteiligung, Intensivstation, oder} Multisystemischem Entzündungssyndrom (MIS-C)

Eine kardiologische Abklärung und Führung dieser Patienten ist durch die Hospitalisation und den schweren Verlauf schon gegeben. Patienten mit MIS-C sollten entsprechend einer Myokarditis im Rahmen einer viralen Erkrankung behandelt werden [22]. In diesem Fall wurde bereits eine kardiologische Abklärung im Akutstadium gemacht. Frühestes Return to Sports nach 3 bis 6 Monaten, wenn sich alle Befunde normalisiert haben [23]. Unabdingbar ist eine Verlaufsuntersuchung mittels EKG, Echo, 24-h-EKG, Belastungstest und je nach Verlauf kardialem MRI zur Bestätigung von normalen Befunden. Auch wenn eine Normalisierung da ist, wissen wir nichts über den Langzeitverlauf von Kindern mit MIS-C, einschliesslich Long-COVID, sodass eine regelmässige weitere Beobachtung durch den Kardiologen als konservative Massnahme durchaus indiziert ist.

\section{Spezialfall: Impfung}

In sehr seltenen Fällen tritt nach Impfung eine Myokarditis auf (in 8-10 Mädchen und bei 56-70 Jungen pro 1 Million Impfdosen) [13]. Die Symptome sind identisch einer infektbedingten Form und treten meist 2-3 Tage nach der 2. Impfung in Form von Thoraxschmerzen auf. Jungen sind zirka 7-mal mehr betroffen als Mädchen, wofür es bisher keine eindeutige Erklärung gibt. Abklärung und Therapie gestalten sind identisch einer infektbedingten Myokarditis, und die Prognose ist sehr gut [13].

\section{Fazit}

Unter Einhaltung von Hygiene- und Distanzregeln sowie dem Fernbleiben vom Sport bei SARS-CoV-2typischen Symptomen können das Entstehen von Infektionsherden und die Übertragung des Coronavirus im Sport minimiert werden, ohne dass die jugendlichen AthletInnen unnötig in ihrer für die physische, 
psychische und soziale Gesundheit essenziellen sportlichen Aktivität eingeschränkt werden. Die Richtlinien für einen optimalen Return to Sports sollen auf dem Schweregrad des Verlaufs basieren. Normalerweise weisen die AthletInnen bei SARS-CoV-2-Infektionen höchstens milde Symptome auf, und der Return to Sports kann problemlos stattfinden. Wegen eines seltenen Risikos einer Begleitmyokarditis im Rahmen einer SARS-CoV-2-Infektion oder als sehr seltene Nebenwirkung der Impfung, welche potenziell zu einem plötzlichen Herztod führen kann, sollten Prävention, Diagnostik und Return to Sports jedoch strikt eingehalten werden.

Acknowledgements, conflict of interest and funding

Wir danken Sidonia Mattmann und Ajay Bharadwaj für ihre wertvolle Hilfe bei der Erstellung des Manuskripts. Es gibt keinen Interessenkonflikt.

\section{Autoren}

Jannos Siaplaouras, Dr. med., praxis@kinderkardiologie-fulda.de

Holger Förster, Dr. med., ordinaation@dr-foerster.at

Christine Joisten, Prof. Dr. med., c.joisten@dshs-koeln.de

\section{Korrespondierende Autorin}

Susi Kriemler, Prof. Dr. med.

Institut für Epidemiologie,

Biostatistik und Prävention (EBPI)

Hirschengraben 84

8001 Zürich

+41446346320

susi.kriemlerwiget@uzh.ch

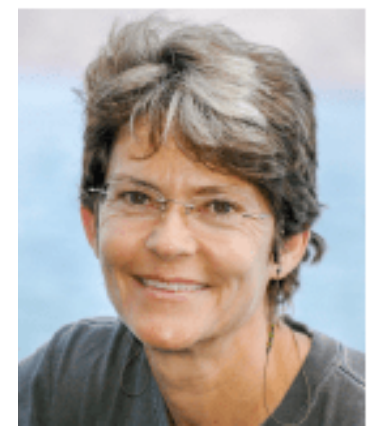

\section{Literatur}

1. Leeb RT, Price S, Sliwa S, et al. COVID-19 Trends Among School-Aged Children - United States, March 1-September 19, 2020. MMWR Morb Mortal Wkly Rep 2020;69(39):1410-15. doi: 10.15585/mmwr.mm6939e2 [published Online First: 2020/10/02].

2. Munblit D, Sigfrid L, Warner JO. Setting Priorities to Address Research Gaps in Long-term COVID-19 Outcomes in Children. JAMA Pediatr 2021 doi: 10.1001/jamapediatrics.2021.2281 [published Online First: 2021/08/03].

3. Osmanov IM, Spiridonova E, Bobkova P, et al. Risk factors for long covid in previously hospitalised children using the ISARIC Global follow-up protocol: A prospective cohort study. The European respiratory journal 2021 doi: 10.1183/13993003.01341-2021 [published Online First: 2021/07/03].

4. Clark JA, Pathan N. Hide and seek in a pandemic: review of SARS-CoV-2 infection and sequelae in children. Experimental physiology 2021 doi: 10.1113/EP089399 [published Online First: 2021/07/10].

5. Xu W, Li X, Dozier M, et al. What is the evidence for transmission of COVID-19 by children in schools? A living systematic review. medR xiv 2020;https://doi.org/10.1101/2020.10.11.20210658. 
6. Ismail SA, Saliba V, Lopez Bernal J, et al. SARS-CoV-2 infection and transmission in educational settings: a prospective, cross-sectional analysis of infection clusters and outbreaks in England. Lancet Infect Dis 2020 doi: 10.1016/S1473-3099(20)30882-3 [published Online First: 2020/12/12].

7. Ulyte A, Radtke T, Abela IA, et al. Evolution of SARS-CoV-2 seroprevalence and 1 clusters in school children from June 2020 to April 2021 reflect community transmission: prospective cohort study Ciao Corona. medRxiv 2021 doi: https://doi.org/10.1101/2021.07.19.21260644.

8. Ulyte A, Radtke T, Abela IA, et al. Clustering and longitudinal change in SARS-CoV-2 seroprevalence in school children in the canton of Zurich, Switzerland: prospective cohort study of 55 schools. Bmj 2021;372:n616. doi: 10.1136/bmj.n616 [published Online First: 2021/03/19].

9. Buitrago-Garcia D, Egli-Gany D, Counotte MJ, et al. Occurrence and transmission potential of asymptomatic and presymptomatic SARS-CoV-2 infections: A living systematic review and metaanalysis. PLoS Med 2020;17(9):e1003346. doi: 10.1371/journal.pmed.1003346 [published Online First: 2020/09/23].

10. COVID-19 Schools infection survey E.

https://wwwonsgovuk/peoplepopulationandcommunity/healthandsocialcare/conditionsanddiseases/b ulletins/covid19schoolsinfectionsurveyround1england/november 2020.

11. L'Huillier AG, Lacour M, Sadiku D, et al. Diagnostic Accuracy of SARS-CoV-2 Rapid Antigen Detection Testing in Symptomatic and Asymptomatic Children in the Clinical Setting. J Clin Microbiol 2021;59(9):e0099121. doi: 10.1128/JCM.00991-21 [published Online First: 2021/07/01].

12. Hause AM, Gee J, Baggs J, et al. COVID-19 Vaccine Safety in Adolescents Aged 12-17 Years - United States, December 14, 2020-July 16, 2021. MMWR Morb Mortal Wkly Rep 2021;70(31):1053-58. doi: 10.15585/mmwr.mm7031e1 [published Online First: 2021/08/06].

13. Bozkurt B, Kamat I, Hotez PJ. Myocarditis With COVID-19 mRNA Vaccines. Circulation 2021;144(6):471-84. doi: 10.1161/CIRCULATIONAHA.121.056135 [published Online First: 2021/07/21].

14. Schwob JM, Miauton A, Petrovic D, et al. Antigen rapid tests, nasopharyngeal PCR and saliva PCR to detect SARS-CoV-2: a prospective comparative clinical trial. medRxiv 2020;https://doi.org/10.1101/2020.11.23.20237057.

15. Phelan D, Kim JH, Chung EH. A Game Plan for the Resumption of Sport and Exercise After Coronavirus Disease 2019 (COVID-19) Infection. JAMA Cardiol 2020 doi: 10.1001/jamacardio.2020.2136 [published Online First: 2020/05/14].

16. Baggish A, Drezner JA, Kim J, et al. Resurgence of sport in the wake of COVID-19: cardiac considerations in competitive athletes. British journal of sports medicine 2020;54(19):1130-31. doi: 10.1136/bjsports-2020-102516 [published Online First: 2020/06/21].

17. Dean P, Jackson L, Paridon S. Returning To Play After Coronavirus Infection: Pediatric Cardiologists` Perspective. Amer Coll Cardiol

2020;https://www.acc.org/latest-in-cardiology/articles/2020/07/13/13/37/returning-to-play-after-corona virus-infection.

18. Pediatrics AAo. COVID-19 Interim Guidance: Return to Sports and Physical Activity. https://servicesaaporg/en/pages/2019-novel-coronavirus-covid-19-infections/clinical-guidance/covid-1 9-interim-guidance- return-to-sports/ 2021.

19. Rajpal S, Tong MS, Borchers J, et al. Cardiovascular Magnetic Resonance Findings in Competitive Athletes Recovering From COVID-19 Infection. JAMA Cardiol 2021;6(1):116-18. doi: 10.1001/jamacardio.2020.4916 [published Online First: 2020/09/12].

20. Clark DE, Parikh A, Dendy JM, et al. COVID-19 Myocardial Pathology Evaluation in Athletes With Cardiac Magnetic Resonance (COMPETE CMR). Circulation 2021;143(6):609-12. doi: 10.1161/CIRCULATIONAHA.120.052573 [published Online First: 2020/12/18].

21. Driggin E, Madhavan MV, Bikdeli B, et al. Cardiovascular Considerations for Patients, Health Care Workers, and Health Systems During the COVID-19 Pandemic. Journal of the American College of 
Cardiology 2020;75(18):2352-71. doi: 10.1016/j.jacc.2020.03.031 [published Online First: 2020/03/24].

22. Canter CE, Simpson KE. Diagnosis and treatment of myocarditis in children in the current era.

Circulation 2014;129(1):115-28. doi: 10.1161/CIRCULATIONAHA.113.001372 [published Online First: 2014/01/08].

23. Maron BJ, Harris KM, Thompson PD, et al. Eligibility and Disqualification Recommendations for Competitive Athletes With Cardiovascular Abnormalities: Task Force 14: Sickle Cell Trait: A Scientific Statement From the American Heart Association and American College of Cardiology. Journal of the American College of Cardiology 2015;66(21):2444-46. doi: 10.1016/j.jacc.2015.09.046 [published Online First: 2015/11/07]. 